\title{
Preparation of Life Science Samples for Energy Dispersive X-ray (EDS) Analysis
}

\author{
Debra M. Sherman
}

Founder and Chief Scientific Officer, DSimaging LLC, Affiliate of the Purdue Research Foundation, West Lafayette, IN 47906, USA

Use of Energy Dispersive X-ray Analysis (EDS), while very common in the materials world, is less often utilized in the life sciences. This is largely due to fewer questions involving these samples that can be answered using elemental analysis. Often presence of toxic amounts of elements can be determined by minimally invasive testing methods (using urine or blood) or chemical analysis of biopsy material. Many of the questions involving abnormal change in biological samples are related to accumulations of fat, proteins and other material in subcellular inclusions. The accumulations are often made up of the common low atomic number elements $(\mathrm{C}, \mathrm{N}, \mathrm{O})$ with various other ions found commonly in such tissues such as $\mathrm{Ca}, \mathrm{Na}, \mathrm{K} \mathrm{P}, \mathrm{Mg}$, etc. These elements are accumulated in relatively small amounts in most biological cells so sufficient amounts over background are often not present. However, there are some cases where amounts are present in sufficient quantity to confirm identity and distribution with EDS, using both overall spectrum and mapping information.

Some examples of biological systems that can benefit from EDX analysis are:

1) Phytoremediation or hyper-accumulation: where plants that are naturally or genetically engineered to accumulate heavy metals, such as Arsenic. ${ }^{1}$

2) Plant biomineralization: formation of crystalline structures within specialized cells in plants.

3) Accumulations of crystals in secretory systems of higher animals and insects such as kidney stones in humans and animals and calcium oxalate crystals in malphighian tubules of insect larvae. $^{2}$

4) Mineral inclusions in cells due to culture/growth conditions. ${ }^{3}$

5) Accumulations of foreign materials in animals such as asbestos and heavy metals from the air, water, or food sources.

6) Localization of tracer materials in organ systems such as those used in diagnostic procedures.

7) Heavy metal tags (colloidal gold, silver) used to identify specific biomarkers such as cell receptors.

The preparation of the samples becomes critical in many of these examples. It is important to assure that mineral or heavy metal deposits are not relocated prior to analysis. Many larger inclusions or deposits are not easily translocated. In this case, routine biological preparation involving chemical fixation and dehydration may suffice. This would be followed by either critical point drying for SEM analysis or embedding and sectioning for TEM analysis. However, even in these cases, it is often desirable to know relative distribution of the inclusions that can be influenced by the inevitable shrinkage during preparation.

CryoSEM is a extremely valuable technique for hydrated samples that allows retention of location in the quickly frozen material (either using plunge or high pressure freezing, HPF) and also enables examination of larger samples than those imaged using TEM. The areas of interest can then be expose by free-fracture, cryo-planing, or FIB. Cryo-sections can also be produced for TEM or SEM analysis although care must be taken to prevent any thawing that would enable relocation of elements. 
Ultrastructure of samples produced using HPF followed by freeze-substitution (FS) for TEM is usually far superior to that from chemically prepared samples although the limited sample size makes location of areas of interest problematic. However, HPF also minimizes the translocation or extraction of elements in the cells during preparation. One example of the severity of this problem is in cyanobacteria where even the very rapid fixation and dehydration using microwave technology removes large amounts of accumulated salts where as these are well preserved using HPF-FS. (Figure 1)

Preparation is quite easy for some lucky researchers using plant tissue. In this case, the rigid cell walls retain the elements within the cells upon simple air-drying of tissue. Simple EDS mapping and analysis as to mineral content can often localize elements accumulated from the soil (Figure 2). More specific localization can be done using TEM following appropriate preparation and thin sectioning.

The most accurate EDX information comes from flat samples where topography does not interfere with $\mathrm{X}$-ray paths to the detector. This may be a problem with some SEM samples where it is not possible to polish or smooth the sample without damaging the desired surface. Other considerations involve attempts to quantify elements in life science samples. Accuracy is hindered by absorption of X-rays from low atomic number elements and reduced pickup of the weaker X-rays by detector components. Lack of suitable standards compound the problem making determination of element amounts using EDS as more of a qualitative rather than quantitative exercise.

References:

1) Pickering et al, Localizing the Biochemical Transformations of Arsenate in a Hyperaccumulating Fern. Environ Sci Technol. 40 (2006) p.5010-5014.

2) Wyman, A.J. and Webb, M.A., Calcium Oxalate accumulation in Malpichian Tubules of Silkworm (Bombyx mori). 1st Annual International Urolithiasis Research Symposium. AIP Conf. Proc. (2006) 900, pp. 407-411

3) Sherman, L.A. and Sherman, D.M. Dept. of Biological Sciences. Purdue University. Personal communication.
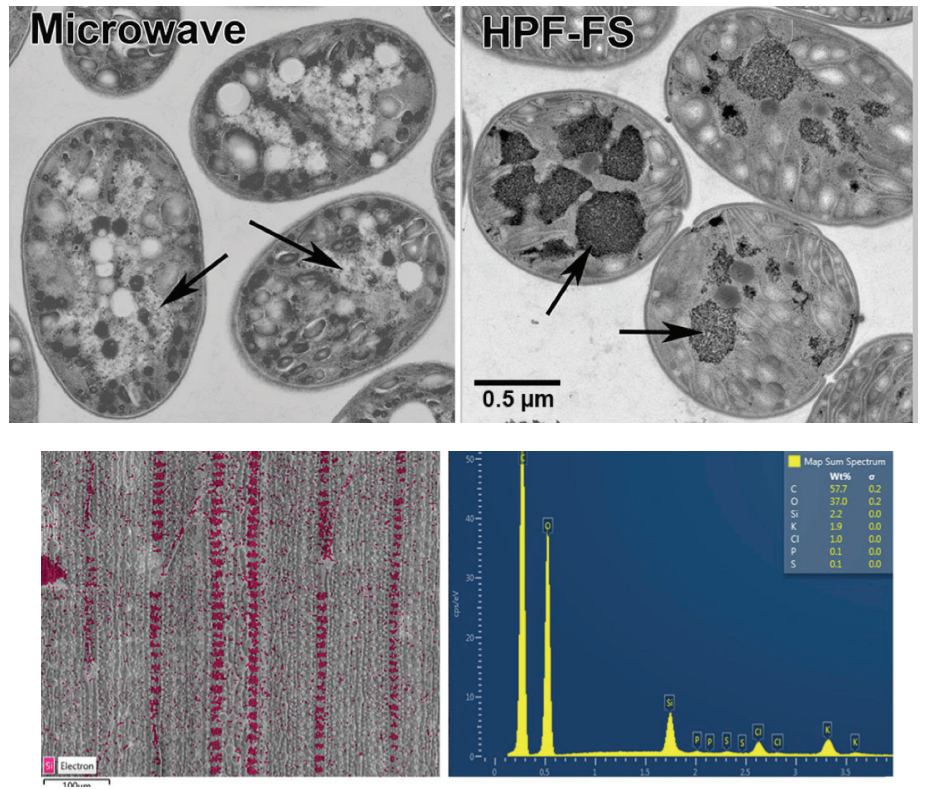

Figure 1: Comparison of ultrastructure in Cyanobacteria Cyanothece Sp. ATCC 51142, flask grown for 10 days in absence of nitrogen, after microwave (MW) and high pressure freeze-freeze substitution (HFP-FS) sample preparation. Note extraction of darker staining mineral deposits in MW cells.

Figure 2: EDX mapping showing localization of Si containing glands in rice leaf prepared by air-drying. 